\title{
No relation between the vertical velocity component and the absolute magnitude among globular clusters
}

\author{
E. A. De Souza-Rossetto and H. J. Rocha-Pinto \\ Observatório do Valongo, Universidade Federal do Rio de Janeiro, Ladeira Pedro Antônio, 43, \\ Saúde, Rio de Janeiro, Brazil \\ email: [erika, helio] @astro.ufrj.br
}

\begin{abstract}
The globular cluster luminosity function distribution shows a peak at $M_{V} \approx-7.5$ mag. There are some indications that the kinematic parameters are correlated with luminosity. In particular, Alfaro et al. (2001) have studied the properties of the Galactic globular cluster system and they found a correlation between spatial-velocity component and globular cluster absolute magnitude. The authors assumed that the globular clusters can be separated into two groups. The first is composed of globular clusters with $M_{V}<-7.5 \mathrm{mag}$ and moving preferentially towards the north Galactic pole, while the faintest globular clusters, composing the second group, move towards the Galactic disk. We have selected a sample of globular clusters using the same criteria as Alfaro et al. (2001) and have checked that this apparent relation indeed exists. Nevertheless, we decided to investigate whether it could be a fortuitous relation or an intrinsic property by checking its validity for eight different epochs at past and future times. The orbital parameters for the globular clusters at these eight epochs were found by orbital integration using a typical Galactic potential. We show that this relation between the vertical velocity component and the absolute magnitude among globular clusters is not coherent with time and the velocity distribution does not support the hypothesis of Alfaro et al. for the existence of two dynamical groups of globular clusters.
\end{abstract}

Keywords. globular clusters: general

\section{Introduction}

The Milky Way hosts around 160 globular clusters (GCs; Harris 1996). The GC system is often divided into subgroups according to parameters like metalicity, spatial distribution, dynamics, kinematics, etc. For example, Hertz \& Grendaly (1983) showed that the GC luminosity function is bimodal. Moreover, Mclaughlin \& Pudritz (1996) have indicated that the GC formation process implies a luminosity function with a characteristic peak for each galaxy type.

van den Bergh (1985) showed that the Galactic GC luminosity function is similar to that of M31, pointing out that the processes that lead to peaks in the distribution should be similar in both galaxies. Also, Capuzzo-Dolcetta (1993) concluded that the evolution of the M31 GC system was strongly affected by dynamical friction and tidal disruption of the GCs near the center.

Based on these assertions, Alfaro et al. (2001) investigated whether it is possible to establish relations between kinematic properties and the luminosity function and, if this were the case, how this could be reflected in the velocity space of GCs. They selected a sample of GCs with proper-motion measurements within a Galactocentric radius of $20 \mathrm{kpc}$. They separated the GCs into two groups: those with $M_{V}$ brighter and fainter 


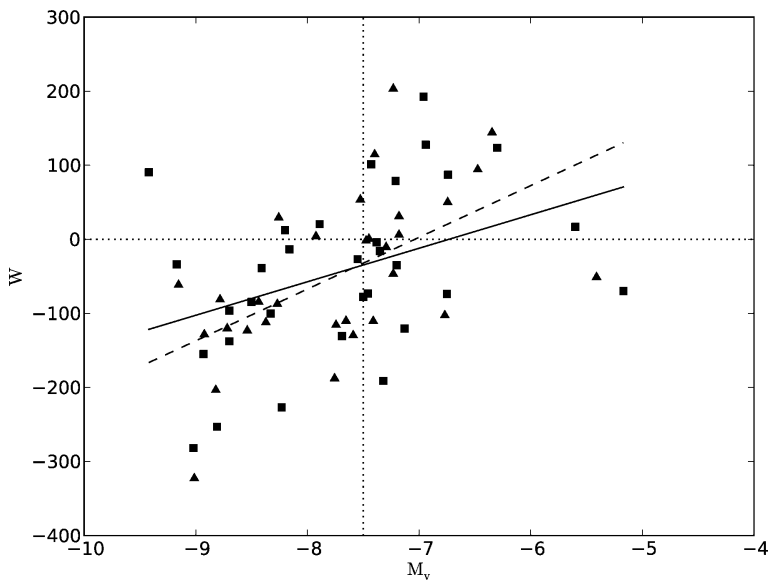

Figure 1. Relation between $W$ and $M_{V}$. The squares and solid line are, respectively, our data and the linear best fit, while the triangles are data from Alfaro et al. (2001) and the dashed line is the best fit to their data.

than $-7.5 \mathrm{mag}$, respectively. The authors found an apparent relation between the vertical velocity component $(W)$ and absolute magnitude $\left(M_{V}\right)$. Also, the brightest GCs in their sample, on average, are moving outwards from the Galactic plane and towards the north Galactic pole. The faintest GCs are characterized by negative motion. To test whether this relation was a selection effect, they estimated the $W$ velocity component and found $\langle W\rangle=-32 \pm 6$ and $46 \pm 6 \mathrm{~km} \mathrm{~s}^{-1}$ for the brightest and faintest clusters, respectively. Based on this, they rejected the selection-effect hypothesis and concluded that the $M_{V}-$ $W$ relation could be primordial. They also found a probability of $<1 \%$ that the two groups of GCs come from the same population.

The goal of this paper is to investigate whether this relation is fortuitous and what its behaviour over the evolution of the Galaxy might be.

\section{A reanalysis of the $M_{V}-W$ relation}

To investigate the relation proposed by Alfaro et al. (2001), we selected a sample of GCs, applying the same criteria used by those authors. We looked for globular clusters with measurements available in the literature of their proper motion, right ascension, declination, distance and radial velocity. We adopted the $M_{V}$ values compiled by Harris (1996). Our final sample contains $44 \mathrm{GCs}$, of which 32 are located within a Galactocentric radius $20 \mathrm{kpc}$. We used the equations of Johnson \& Soderblom (1987) to calculate the spatial velocity components, $U, V$ and $W$ in a left-handed system, with the $U$ component pointing from the Sun to the Galactic Center. We corrected for solar motion using $(u, v, w)_{\odot}=(-9,11,6)$ and $\Theta_{0}=220 \mathrm{~km} \mathrm{~s}^{-1}$.

In Figure 1 we show the $W-M_{V}$ plot for our (squares) and Alfaro et al. (2001)'s (triangles) samples. The solid and dashed lines are, respectively, our and Alfaro et al. (2001)'s linear fits. Our data show that this relation is much less defined than in Alfaro et al. (2001), to the point of rendering it doubtful. Take, for instance, the much larger numbers of bright GCs characterized by negative $W$ that were not seen in Alfaro et al. (2001). Nevertheless, a relation does indeed exist. 


\section{Temporal evolution of the $M_{V}-W$ relation}

Despite having found a similar relation as Alfaro et al. (2001), we decided to investigate whether it remains coherent over time. For this purpose, we used the Galactic potential proposed by Flynn et al. (1996). We integrated this potential and calculated the $W$ velocities for eight different epochs from $8 \mathrm{Gyr}$ ago until $8 \mathrm{Gyr}$ in the future, at intervals of 2 Gyr. For each specific epoch, we analysed the relation between $W$ and $M_{V}$. Moreover, we checked whether the brightest and faintest globular clusters are separated into two kinematical groups using by a $\mathrm{K}-\mathrm{S}$ test. For the current epoch, providing support to Alfaro et al. (2001)'s claim, we find a probability of 0.074 that the GCs are from the same sample. However, for others epochs, this probability does not support this interpretation. In Figure 2 we plot the $W-M_{V}$ diagrams for the eight epochs analysed. The panels in this figure show that the GCs mix and the relationship between $W$ and $M_{V}$ changes considerably. Analysing the evolution of the relation, we conclude that the present-day $W-M_{V}$ relation is an artifact of the present-day kinematical configuration of the GC system.

\section{Conclusions}

We have found a similar relation between $W$ and $M_{V}$ for the current epoch among GCs as proposed by Alfaro et al. (2001). However, analysing the past and the future analogues of this relation, we conclude that the hypothesis presented by these authors is not valid. In others words, there is no physical relation between $W$ and $M_{V}$ for GCs and the GCs cannot be separated into two distinct groups according to their $W$ velocity distribution.
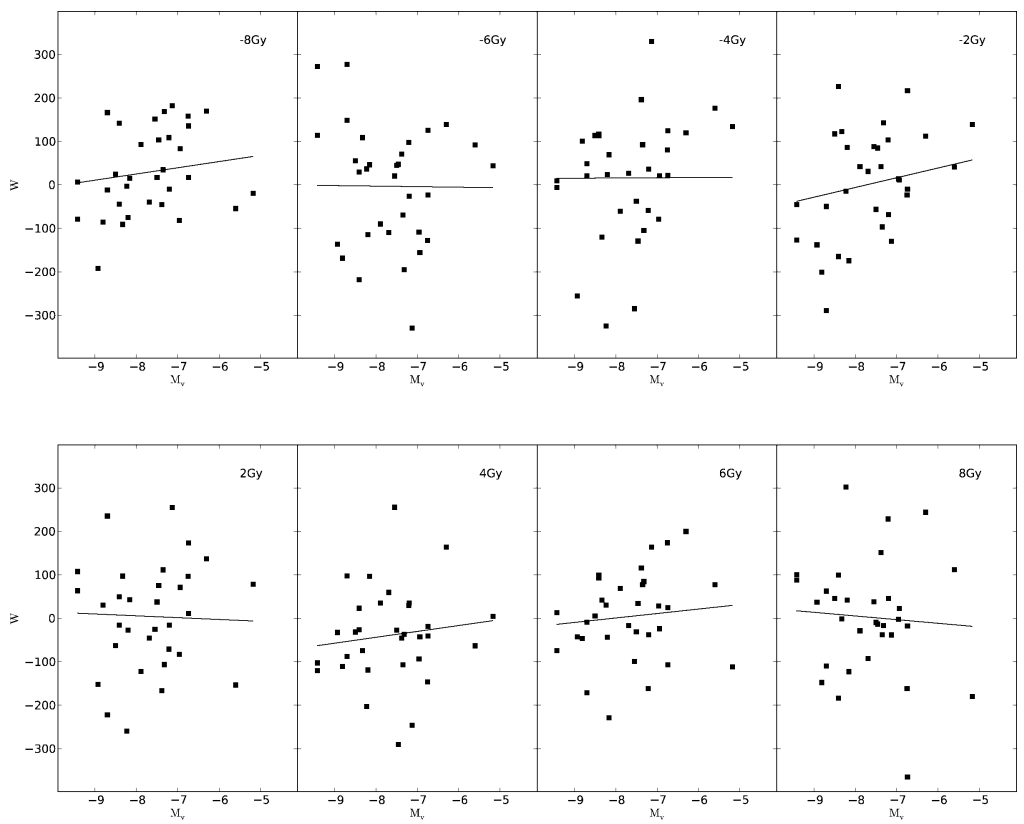

Figure 2. Relation between $W$ and $M_{V}$ for eight different epochs. We see that the GCs are mixing randomly. As a consequence, the relation between $W$ and $M_{V}$ does not fluctuate periodically, so that the relation is not primordial as proposed by Alfaro et al. (2001). 


\section{References}

Alfaro, E. J., Delgado, A. J., Gómes-Flechoso, M. A., Ferrini, F., \& Castro, I. 2001, A\&A, 370, 45

Capuzzo-Dolcetta, R. 1993, ApJ, 415, 616

Flynn, C., Sommer-Larsen, J., \& Christensen, P. R. 1996, MNRAS, 281

Johnson, D. R. H. \& Soderblom, D. R. 1987, AJ, 93, 864

Hertz, P. \& Grendaly, J. 1983, ApJ, 275, 105

Mclaughlin, D. E. \& Pudritz, R. E. 1996, ApJ, 475, 578

van den Bergh, S. 1985, ApJ, 297, 361

Harris, W. E. 1996, AJ, 112, 1487 